\title{
Electromagnetic-wave propagation through dispersive and absorptive photonic-band-gap materials
}

\author{
M. M. Sigalas, C. M. Soukoulis, C. T. Chan, and K. M. Ho \\ Ames Laboratory and Department of Physics and Astronomy, Iowa State University, Ames, Iowa 50011
}

(Received 10 January 1994)

\begin{abstract}
Using the transfer-matrix method, we calculate the transmission coefficient versus the frequency of the incident electromagnetic waves propagating in photonic-band-gap structures constructed from dispersive and highly absorbing materials. We study how the photonic band gaps are affected by the presence of polariton gaps and/or absorption. Also, the possible difficulties of their experimental investigation are discussed.
\end{abstract}

\section{INTRODUCTION}

The problem of the electromagnetic (EM) wave propagation in periodic and/or random dielectric structures has received increasing interest in recent years. The appearance of forbidden frequency regions - the so-called photonic band gaps (PBG) - in the spectrum of EM waves propagating in such composite dielectric materials can have potential applications ${ }^{1,2}$ in several scientific and technical areas such as antennas, filters, optical switches, design of more efficient lasers, etc. It is, therefore, very important to find three-dimensional (3D) and twodimensional (2D) dielectric structures possessing PBG. Theoretical calculations of Ho, Chan, and Soukoulis in 3D have shown that periodic dielectric materials with diamond $^{3}$ or diamondlike structures ${ }^{4}$ can indeed have PBG. One of these structures, the so-called "3-cylinder" structure, which consists of three sets of cylinders drilled along the (110), (101), and (011) directions at each point of a fcc lattice, has been fabricated ${ }^{5}$ in the millimeter length scale and shown to possess full PBG in the microwave region in agreement with the theoretical predictions. ${ }^{4}$ Very narrow PBG's have been also found for simple cubic structures. ${ }^{6}$ Similar calculations in 2D structures $^{7-9}$ have shown that a triangular lattice of air columns in a dielectric background is the best overall $2 \mathrm{D}$ structure, which gives the largest PBG with the smallest index contrast. As in the case of electrons, lattice imperfections in both 2D and 3D periodic dielectric structures can create fully localized EM wave functions. ${ }^{10-14}$ Most of the fabricated composite dielectric materials ${ }^{10,12,13}$ exhibit PBG's at microwave frequencies, since the fabrication of ordered dielectric structures in optical length regions is more difficult. However, the creation of such structures in the optical wavelength region is the most challenging prospect in that area. ${ }^{1,2}$

Most of the theoretical calculations ${ }^{1-9}$ take into account the similarity that exists between electronic states in a periodic potential and the EM waves in a periodic dielectric medium. Techniques, such as the plane-wave method (PWM) developed for the electronic case, have been successfully applied to calculate the band structure of EM waves propagating in either $2 \mathrm{D}$ or $3 \mathrm{D}$ periodic dielectric structures. Using the PWM, the dispersion of the photon bands in an infinite periodic structure can be calculated; in other words, the eigenfrequencies $\omega(\mathbf{k})$ are calculated for each real wave vector $\mathbf{k}$. An experimental investigation focuses mainly on the transmission of EM waves through a finite slab of a PBG material patterned in the required periodic structure; thus, the comparison with the results from the PWM is a nontrivial task. Also, when the dielectric constant $\epsilon$ is frequency dependent or when it has a non-negligible imaginary part, the PWM is not useful. Recently, Pendry and MacKinnon ${ }^{14}$ introduced a complementary technique of studying PBG structures. This is essentially a transfer-matrix method (TMM) which has the advantage that the transmission and reflection coefficients for incident EM waves of various frequencies can be obtained directly from the calculations. The TMM can also be efficiently used in cases where the $\epsilon$ is frequency dependent, or it has a large imaginary part. Disordered systems and periodic systems with imperfections can also be studied using the TMM. ${ }^{15}$ Finally, the band structure of an infinite periodic system can be found using the TMM, but this method calculates all the bands $\mathbf{k}(\omega)$ for a given $\omega$.

As we stated previously, the creation of PBG materials in the infrared and the optical wavelength regions is still an open and very challenging problem. However, in that region some materials have frequency-dependent dielectric constants or they are highly absorbing.

In this paper we study how these two properties affect the EM waves propagating in periodic or disordered structures. We calculate the transmission of EM waves versus frequency using the TMM. In Sec. II, we study cases where the structural gap, which is created by the periodicity of the lattice, is close to the polariton gap (PG), which is produced by the coupling between the EM waves and the transverse optical phonons. In Sec. III, we study how the structural gap is affected when the EM waves are propagated in highly absorbing PBG materials. GaAs is used as a prototype material because it has already been tried, without success, for the developing of PBG materials with gaps into the infrared region. ${ }^{16}$ However, the same conclusions can be reached for other materials with a similar behavior. 


\section{POLARITON VS STRUCTURAL GAP}

It is well known that the coupling between the transverse optical phonons and the transverse EM waves entirely changes the character of the propagation of EM waves. This coupling can be described by the following frequency dependent dielectric constant: ${ }^{17}$

$$
\epsilon(\omega)=\epsilon_{\infty}+\left(\epsilon_{0}-\epsilon_{\infty}\right) \frac{\omega_{T}^{2}}{\omega_{T}^{2}-\omega^{2}-i \Gamma \omega},
$$

where $\epsilon_{0}, \epsilon_{\infty}$ are the dielectric constants for $\omega=0$ and $\infty$, respectively, $\omega_{T}$ is the transverse optical phonons frequency, and $\Gamma$ is the absorption coefficient. For $\Gamma=0$, we can write Eq. (1) in a more familiar form:

$$
\epsilon(\omega)=\epsilon_{\infty} \frac{\omega_{L}^{2}-\omega^{2}}{\omega_{T}^{2}-\omega^{2}},
$$

where $\omega_{L}^{2}=\omega_{T}^{2} \epsilon_{0} / \epsilon_{\infty}$. The $\epsilon(\omega)$ for GaAs is shown in Fig. 1, using the following parameters: $\epsilon_{\infty}=10.9$, $\omega_{L}=8.75 \mathrm{THz}$, and $\omega_{T}=8.12 \mathrm{THz}{ }^{17}$ Dielectric constants described by Eq. (2) create a forbidden band (polariton gap) between the frequencies $\omega_{L}$ and $\omega_{T}$ which has nothing to do with the periodicity of the lattice.

In Figs. 2(a)-2(c), the transmission vs the frequency is plotted for EM waves propagating in a 2D square lattice consisting of GaAs cylinders with filling ratio $(f=0.449)$ embedded in air; the EM waves are $E$-polarized ( $E$-field is parallel to the axis of the cylinders). The dashed lines in Figs. 2(a)-2(c) correspond to cases where the GaAs has frequency independent dielectric constant $\left(\epsilon=\epsilon_{\infty}\right)$, while the solid lines correspond to cases where $\epsilon(\omega)$ for GaAs are described by Eq. (2). For lattice constant $a=5.655$ $\mu \mathrm{m}$ [Fig. 2(a)], the first structural gap is above the polariton gap [vertical dotted lines in Figs. 2(a)-(2c)]; for $a=7.540 \mu \mathrm{m}$ [Fig. 2(b)], the two gaps are overlapping, while for $a=9.425 \mu \mathrm{m}$ [Fig. 2(c)] the first structural gap is below PG and the second structural gap is above PG. In all the cases, there is a huge drop in the transmission inside the PG (the light is totally reflected) as in the homogeneous case $(f=1)$. This drop is strongly affected by the filling ratio of GaAs and, as we expected, the drop tends to disappear as $f \rightarrow 0$. But the most interesting result is the appearance of new gaps in the frequencydependent dielectric constant case. For $a=5.655 \mu \mathrm{m}$ [solid line in Fig. 2(a)], there are two gaps (in addition to the polariton gap) with midgap frequencies $\omega_{g}=7.7$ and $12 \mathrm{THz}$, while for $\epsilon=\epsilon_{\infty}$ there is only one gap at $\omega_{g}=11$ $\mathrm{THz}$ [dashed line in Fig. 2(a)]. The situation is nearly the same for $a=7.540 \mu \mathrm{m}$ with differences regarding the size of the two gaps. In contrast, for $a=9.425 \mu \mathrm{m}$ there are two relatively wide gaps with $\omega_{g}=5.8$ and $13.5 \mathrm{THz}$, and two smaller gaps with $\omega_{g}=7.7$ and $9.4 \mathrm{THz}$ [solid line in Fig. 2(c)]; notice that the second structural gap is closer to the PG as compared with the other cases. In the following paragraph, we will try to explain these differences by using a multistep dielectric constant as an approximation to Eq. (2).

Roughly speaking, the conditions for the appearance of the gaps can be described by the relation

$$
\frac{\omega_{g}^{n} a \sqrt{\bar{\epsilon}}}{c_{0}}=k_{n}, \quad n=1,2,3, \ldots,
$$

where $c_{0}$ is the velocity of light in the air, $\bar{\epsilon}$ is the average value of the dielectric constant over the space, $\omega_{g}^{n}$ is the midgap frequency of the $n$th gap, $k_{n}$ is a constant characterizing the $n$th gap, and $a$ is the lattice constant. Knowing the constant $k_{n}, \bar{\epsilon}$, and $a$, we can approximately estimate the frequency, $\omega=\omega_{g}^{n}$, in which the $n$th gap will appear. For the polariton case, we can approximate the dielectric constant described by Eq. (2) with a multistep dielectric function (see the dashed line in Fig. 1):

$$
\epsilon(\omega) \simeq\left\{\begin{array}{l}
13.26, \quad \omega<6 \mathrm{THz} \\
20.47, \quad 6 \mathrm{THz}<\omega<8.12 \mathrm{THz} \\
-60.00, \quad 8.12 \mathrm{THz}<\omega<8.75 \mathrm{THz} \\
6.54, \quad 8.75 \mathrm{THz}<\omega<11 \mathrm{THz} \\
10.13, \quad 11 \mathrm{THz}<\omega,
\end{array}\right.
$$

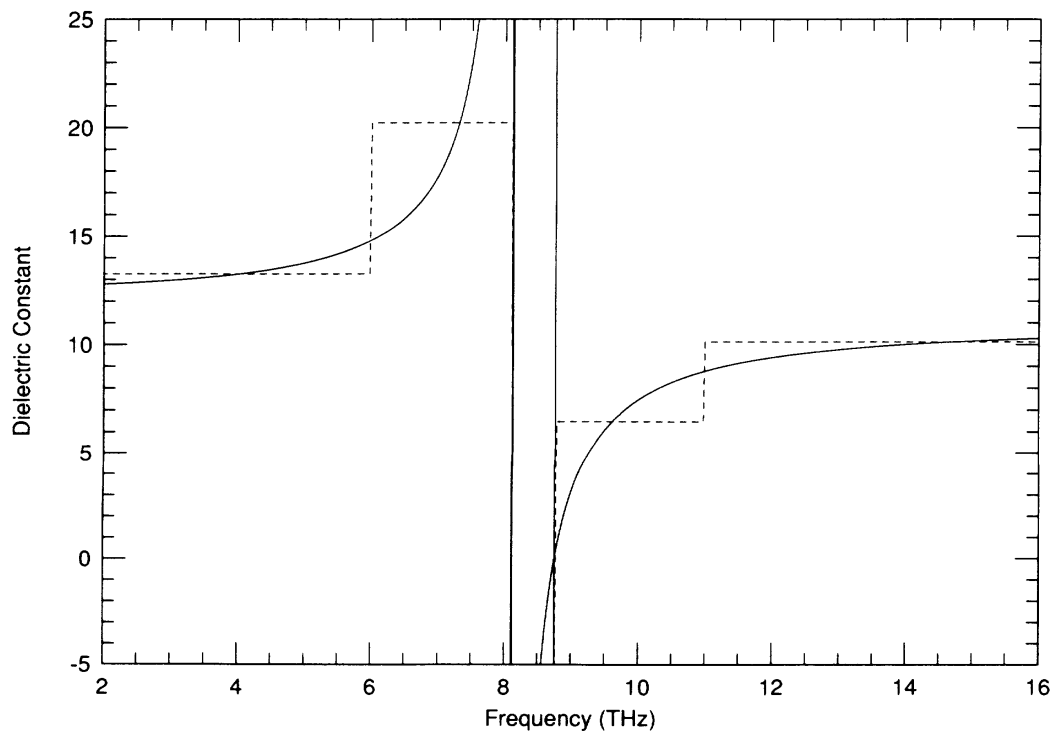

FIG. 1. The dielectric constant of GaAs as given by Eq. (2) (solid line) and its approximate value (dashed line) as described by Eq. (4). 

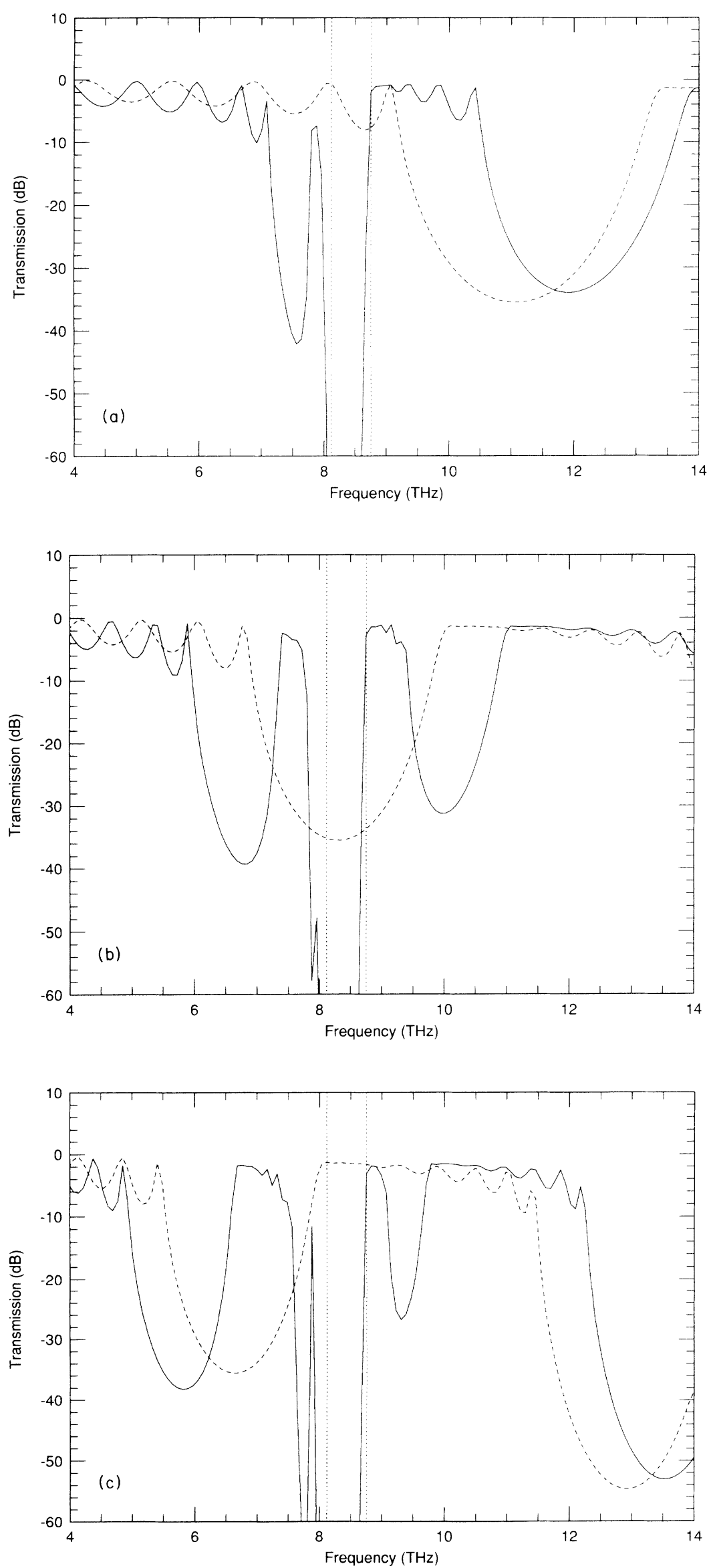

FIG. 2. The transmission vs the frequency for $E$-polarized EM waves propagating in a perfect $2 \mathrm{D}$ square lattice consisting of nonabsorbing $(\Gamma=0) \mathrm{GaAs}$ cylinders with $f=0.449$ lattice constant $a=5.655 \mu \mathrm{m}$ (a), $7.540 \mu \mathrm{m}(\mathrm{b})$, $9.425 \mu \mathrm{m}$ (c), and the slab thickness is $8 a$ for all the cases. The dielectric constant of GaAs is given by Eq. (2) (solid lines) or it is assumed constant $\epsilon=\epsilon_{\infty}$ (dashed line). The vertical dotted lines are the limits of the PG. 
where the approximate values of $\epsilon(\omega)$ have been calculated by averaging $\epsilon^{-1}(\omega)$ (for $\omega<8.12 \mathrm{THz}$ ) or $\epsilon(\omega)$ (for $\omega>8.12 \mathrm{THz}$ ) over the certain frequency regions. Using the previous averaging procedure, we get the best overall agreement between the multistep model and the results from Eq. (2) (compare solid and dashed lines in Fig. 1). Also, by averaging the dielectric constant given by Eq. (4) over the volume, we find

$$
\bar{\epsilon}(\omega) \simeq\left\{\begin{array}{l}
6.505, \quad \omega<6 \mathrm{THz} \\
9.742, \quad 6 \mathrm{THz}<\omega<8.12 \mathrm{THz} \\
-26.389, \quad 8.12 \mathrm{THz}<\omega<8.75 \mathrm{THz} \\
3.488, \quad 8.75 \mathrm{THz}<\omega<11 \mathrm{THz} \\
5.099, \quad 11 \mathrm{THz}<\omega
\end{array}\right.
$$

where for the frequency independent case $\left(\epsilon=\epsilon_{\infty}\right)$ $\bar{\epsilon}=5.445$ for $f=0.449$. Using the previous value for $\bar{\epsilon}$, we can find from Eq. (3) with the help of Fig. 2 that $k_{1}=0.48$. We are now able to calculate the predicted values of $\omega_{g}^{1}$ from our multistep model [Eq. (5)].

For $\omega>11 \mathrm{THz}$ and $a=5.655 \mu \mathrm{m}$, the multistep model predicts $\omega_{g}^{1}=11.4 \mathrm{THz}$, which is close to the value of $\omega_{g}$ calculated from the TMM $\left(\omega_{g} \simeq 12 \mathrm{THz}\right)$. But more interesting is that this simplified model predicts that the conditions for the appearance of the first gap can be also fulfilled in the region $6 \mathrm{THz}<\omega<8.12 \mathrm{THz}$. In particular, the model predicts that $\omega_{g}^{1}=8.2 \mathrm{THz}$ and, as we can see from Fig. 2(a) for the TMM calculation, there is a gap in that region with $\omega_{g}^{1}=7.5$. The validity of the multistep model is further supported from similar comparisons for $a=7.540 \mu \mathrm{m}$.

From the previous arguments, we can reach the conclusion that for frequency dependent dielectric constants described by Eq. (2), gaps can be found at two different frequency regions. For this reason, we call them "twin" gaps. The concept of "twin" gaps is further supported by the results presented in Fig. 2(c) for $a=9.425 \mu \mathrm{m}$. Using the value of $k_{1}=0.48$ and Eq. (3), the multistep model predicts that the first gap will appear in two different frequency regions with $\omega_{g}^{1}=5.4$ and $8.7 \mathrm{THz}$, while the TMM gives $\omega_{g}^{1}=5.7$ and $9.1 \mathrm{THz}$. For the second gap $k_{2}=0.96$, so the multistep model predicts "twin" gaps with $\dot{\omega}_{g}^{2}=7.3$ and $13.9 \mathrm{THz}$, which are very close to the calculated values from the TMM $\left(\omega_{g}^{2}=7.8\right.$ and 13.5 THz). TMM calculations with smaller frequency steps around the PG, where the dielectric constant changes rapidly, have shown there are additional sharp drops in the transmission which, according to the previous prescription, are the "twin brothers" of the other higher gaps.

Another consequence of the frequency dependence of the dielectric constant is the narrowing of the gaps. In particular, for $a=5.655 \mu \mathrm{m}$ the widths of the first gap over the midgap frequency $\left(\Delta \omega / \omega_{g}\right)$ for a dielectric function $\epsilon$ described from Eq. (2) [solid line in Fig. 2(a)] are 0.286 and 0.108 for each of the "twin" gaps, which are smaller as compared with the $\Delta \omega / \omega_{g}(0.386)$ for constant $\epsilon=\epsilon_{\infty}$ [dashed line in Fig. 2(a)]. Also, as we can see in Figs. 2(a) -2 (c), for the other two lattice constants, the gaps which are closer to the PG are narrower than their "twin" gaps. Since the dielectric constant [Eq. (2)] changes rapidly for $\omega$ around the PG, the bands directly above the certain gap "see" higher (or lower) dielectric constants as compared with the bands below the gap, depending on the location of the gap relative to the PG. As a consequence of this, the higher (or lower) bands move to lower (or higher) frequencies; thus, the gap becomes smaller. Notice there is an additional reason which is responsible for the differences between the frequencydependent and the frequency-independent dielectric constant cases: For the frequency-independent case, we assume $\epsilon=\epsilon_{\infty}=10.9$, which is always higher (smaller) than the frequency-dependent dielectric constant [Eq. (2)] for frequencies above (below) the PG, so the gaps will appear in slightly smaller (higher) frequencies for the $\epsilon=\epsilon_{\infty}$ cases [see Figs. 2(a)-2(c)].

The concept of the "twin" gaps is quite general and it appears for any kind of structure in one-, two-, and three-dimensional systems. However, for the 2D lattice consisting of GaAs cylinders embedded in air, the EM waves with frequencies inside the PG exhibit different behavior depending on the polarization of the waves. For $E$ parallel to the axis of the cylinders, the wave is actually totally reflected, since the transmission is very small inside the PG region (see Fig. 2). On the other hand, for $E$ perpendicular to the axis of the cylinders, there are frequencies inside the PG region where there is transmission. We can compare the present case with what happens in a system consisting of infinite conducting wires forming a 2D lattice [the dielectric constant of the wires described by $\epsilon(\omega)=1-\omega_{p}^{2} / \omega^{2}$, where $\omega_{p}$ is the plasma frequency] for $\omega<\omega_{p}$; in both cases, the dielectric constant is negative. It is well known that the conducting wires system can be used as a polarizer, since the waves with $E(H)$ parallel to the axis of the cylinders are almost totally reflected (transmitted).

The transmission versus frequency is shown in Fig. 3, for a case where two second neighboring cylinders have been removed from an otherwise perfect $2 \mathrm{D}$ square lattice consisting of $6 \times 6 \mathrm{GaAs}$ cylinders with $f=0.449$ embedded in air; the EM waves are $E$-polarized and the lattice constant is $a=9.425 \mu \mathrm{m}$. As we can see in Fig. 3, each of the "twin" gaps has a different behavior regarding the appearance of the localized states inside the gaps. In particular, for the first gap around $6 \mathrm{THz}$, there are several localized states close to the lower edge of the gap (see the solid line in Fig. 3) which do not appear in its "twin" gap at around $9.5 \mathrm{THz}$; but the localized states in the middle of the gap are a common feature for both "twins." Also, for both "twins," the transmission close to the upper edge of the gaps is nearly the same as in the periodic case (dashed line in Fig. 3). The differences are more obvious for the two gaps which correspond to the second structural gap. For the gap at around $13.5 \mathrm{THz}$, there are two well-defined localized states at $\omega=14$ and 14.2 THz, while its "twin" gap at $\omega=7.75 \mathrm{THz}$ is nearly unaffected from the disordering procedure, as we can see from the transmission, which is nearly the same for both the disorder (solid line in Fig. 3) and the periodic (dashed line in Fig. 3) cases. It seems that for the gaps which lie close to the PG-where the $\epsilon(\omega)$ change rapidly - the 


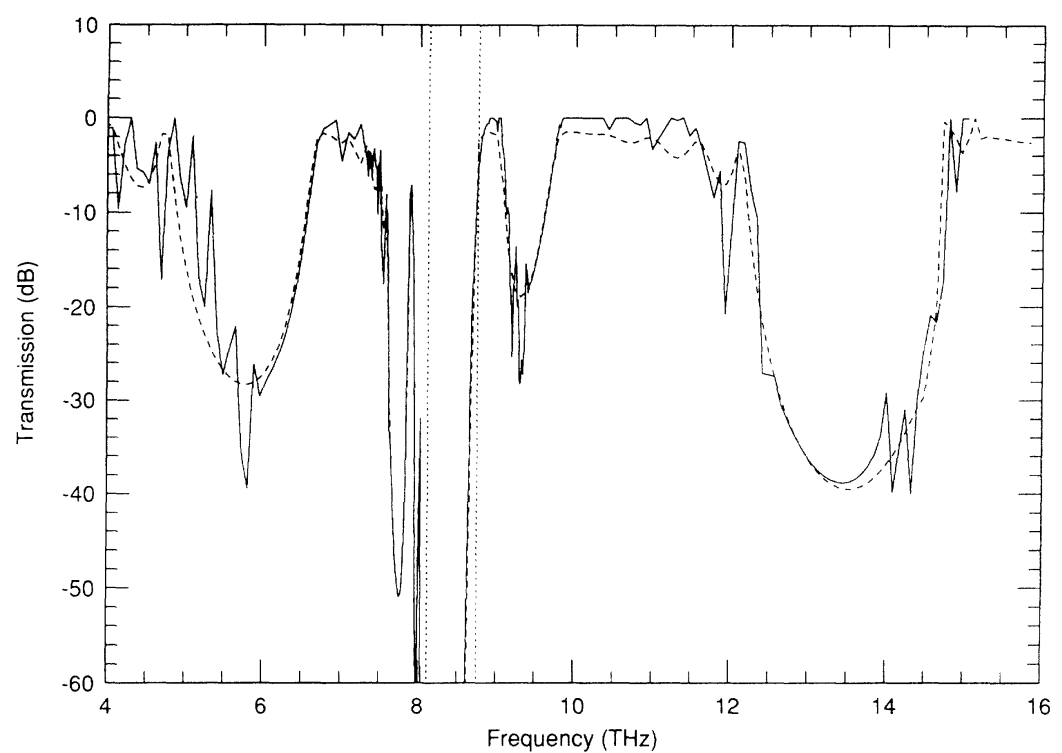

FIG. 3. The transmission vs the frequency for $E$-polarized EM waves propagating in a 2D square lattice consisting of $6 \times 6$ nonabsorbing $(\Gamma=0)$ GaAs cylinders with $f=0.449$, $a=9.425 \mu \mathrm{m}$. The dashed line corresponds to the perfect lattice while the solid line corresponds to the case where two secondneighboring cylinders have been removed. defect states are more well localized and one expects the localization length to be smaller in that frequency region.

In all the previous cases, we have assumed nonabsorbing materials $[\Gamma=0$ in Eq. (1)]. But, frequency-dependent dielectric constants are always accompanied by rather high absorption. In Fig. 4, the transmission vs the frequency is shown for a 2D square lattice consisting of GaAs cylinders with $f=0.449$. The EM waves are $E$ polarized, $a=7.540 \mu \mathrm{m}$, and the thickness of the slab is $8 a$. The dielectric constant of GaAs is described by Eq. (1) with $\Gamma=0.5$ and $0.1 \mathrm{THz}$. The value $\Gamma=0.5 \mathrm{THz}$ fits fairly well with the experimental values for the dielectric constant of GaAs. ${ }^{18}$ For small absorption $(\Gamma=0.1 \mathrm{THz}$; dashed line in Fig. 4), the results are comparable to the nonabsorbing case [Fig. 2(b)] except for a small drop in the transmission around the PG. At $\omega=7.5 \mathrm{THz}$, the transmission for $\Gamma=0.5 \mathrm{THz}$ is $-35 \mathrm{~dB}$, while for $\Gamma=0.1 \mathrm{THz}$ it is only $-10 \mathrm{~dB}$. This strong drop in the transmission for $\Gamma=0.50 \mathrm{THz}$ makes the experimental investigation of the "twin" gaps difficult because of the drop due to the absorption becomes comparable to the drop due to the periodicity of the structure. For even higher $\Gamma$, the "twin" gaps and the PG become one wide gap because there is no recovering of the transmission at edges of the "twin" gaps which are closer to the PG.

\section{HIGHLY ABSORBING PBG STRUCTURES}

In the optical wavelength region, the dielectric constant of most of the materials has an appreciable imaginary part. In the following section, we examine how the gaps are affected by the absorption. We choose GaAs as

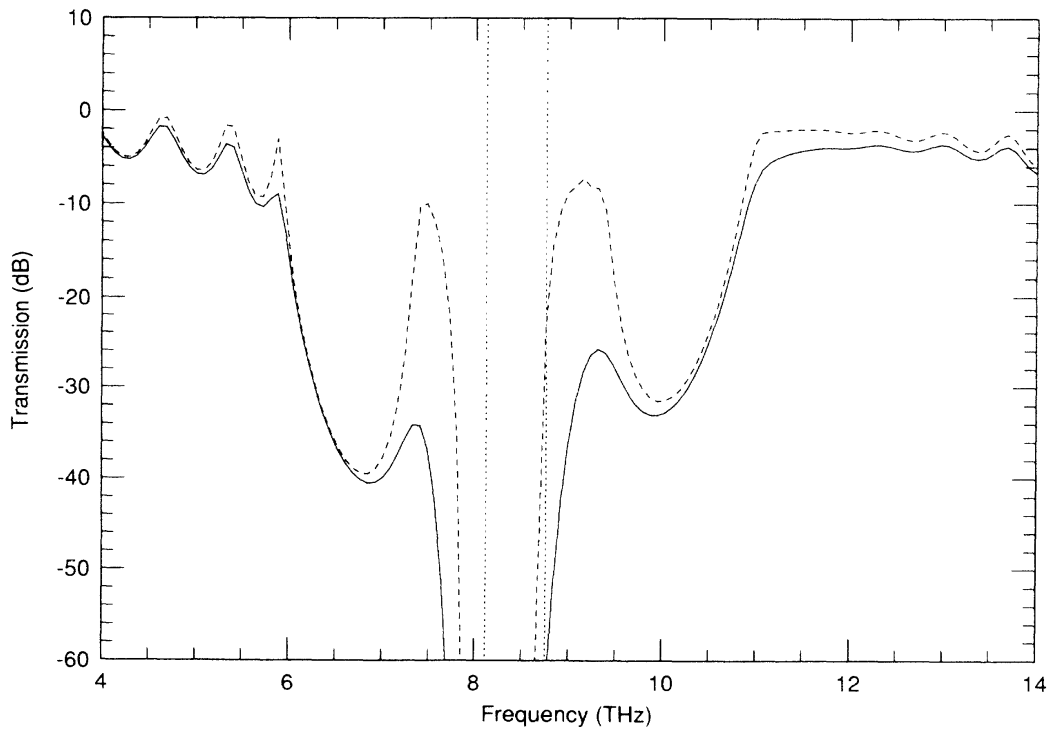

FIG. 4. The transmission vs the frequency for the same case as in Fig. 2(b) with $\Gamma=0.1$ $\mathrm{THz}$ (dashed line) or $0.5 \mathrm{THz}$ (solid line). 


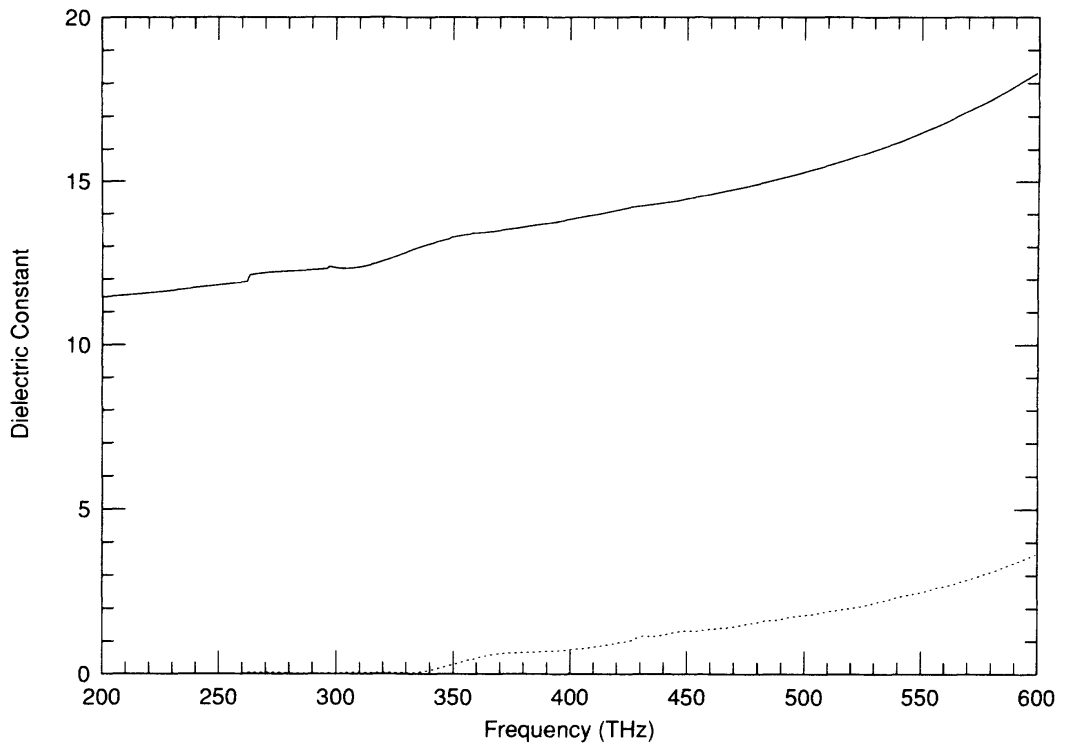

FIG. 5. The experimental values of the real (solid line) and imaginary (dashed line) part of the dielectric constant of GaAs as given in Ref. 18. a PBG material, since it is a possible candidate material for the development of PBG "crystals" in the optical wavelength region, although the same conclusions can be reached for other absorbing materials. Recently, Yablonovitch ${ }^{17}$ tried to develop a PBG "crystal" consisting of an array of microscopic crisscrossing holes, $0.5 \mu \mathrm{m}$ in diameter and $0.7 \mu \mathrm{m}$ apart, drilled into GaAs; this "crystal" has the "3-cylinder" structure described previously. His trial was not successful, since no transmission data were shown with a true gap. Theoretically, the first gap is expected to be between 200 and $270 \mathrm{THz}$, which is in the infrared region. As we can see from Fig. 5, the imaginary part of the dielectric constant is negligible in that region; but, as the frequency approaches the optical region, the imaginary part of the dielectric constant increases and it becomes appreciable above $340 \mathrm{THz}$.

Using the experimental values of the GaAs dielectric constant, ${ }^{18}$ we have calculated the transmission vs the frequency for three different lattice constants, $a=0.4$, 0.5 , and $0.6 \mu \mathrm{m}$ (Fig. 6). In all cases the structure is the "3-cylinder" structure with air holes in GaAs; the filling ratio of GaAs is 0.221 and the thickness of the slab is $4 a$. For $a=0.6 \mu \mathrm{m}$ (dotted line in Fig. 6) the first gap appears between 270 and $330 \mathrm{THz}$; since the imaginary part of $\epsilon$ is negligible in that region, the gap is not affected by the absorption. But above $340 \mathrm{THz}$, there is a continuous drop of the transmission as a result of the increase of the imaginary part of $\epsilon$. For $a=0.5 \mu \mathrm{m}$ (dashed line in Fig. 6), the first gap starts to become affected by the absorption. The transmission at the upper edge of the gap is $-20 \mathrm{~dB}$ while for $a=0.6 \mu \mathrm{m}$ it is $-2 \mathrm{~dB}$. The effect of the absorption is even stronger for $a=0.4 \mu \mathrm{m}$ (solid line in Fig. 6); in that case, the transmission at the upper edge of the gap is $-36 \mathrm{~dB}$, while it is $-44 \mathrm{~dB}$ in the center of the gap; thus, the drop due to the absorption becomes comparable with the drop of the transmission due to the underlying structure. For even smaller lattice constants there is no recovery of the transmission at the upper edge of the gap and the transmission is continuously dropping. So, one must be very careful in designing photonic band

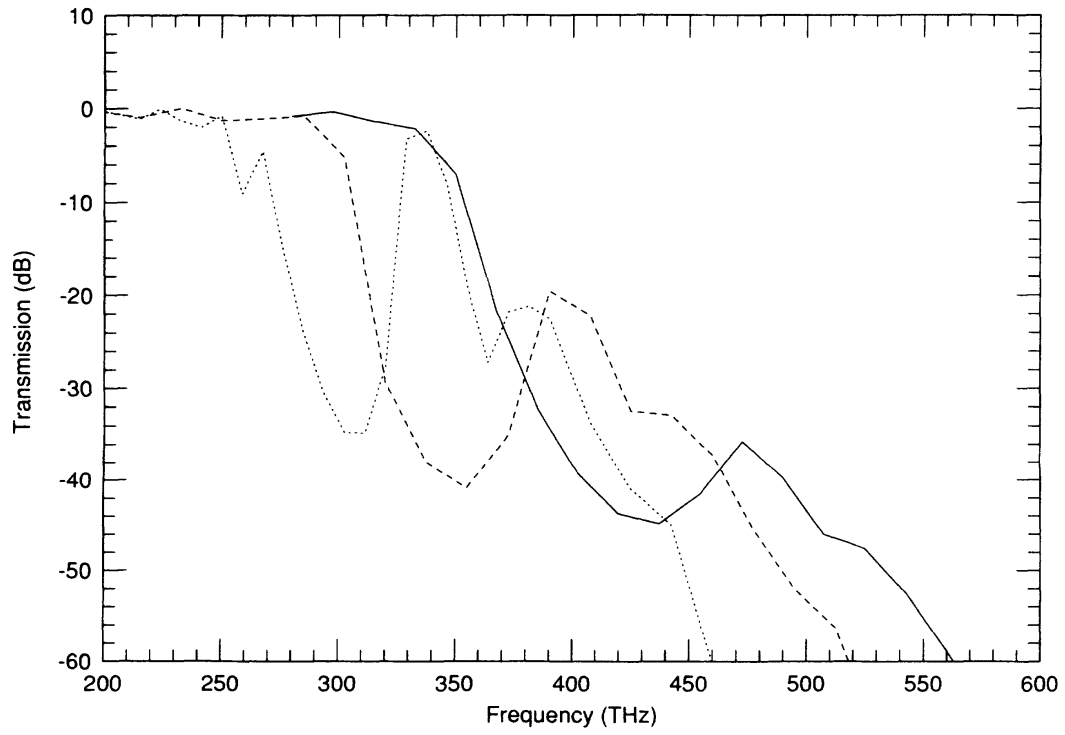

FIG. 6. The transmission vs the frequency for EM waves propagating along the (001) direction in a "3-cylinder" structure constructed of GaAs with $f=0.221$ and lattice constants $a=0.4 \mu \mathrm{m}$ (solid line), $0.5 \mu \mathrm{m}$ (dashed line), and $0.6 \mu \mathrm{m}$ (dotted line). The slab thickness is $4 a$. 


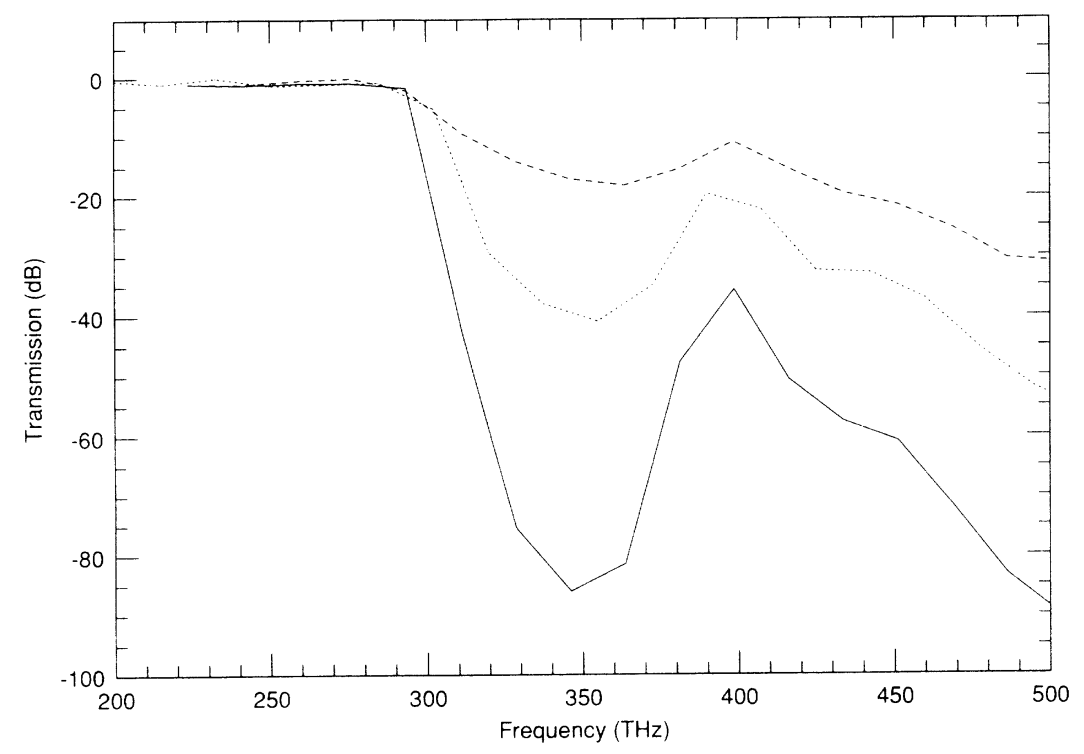

FIG. 7. The transmission vs the frequency for EM waves propagating along the (001) direction in a "3-cylinder" structure constructed of GaAs with $f=0.221$ and lattice constant $a=0.5 \mu \mathrm{m}$. The slab thickness is $2 a$ (dashed line), $4 a$ (dotted line), and $8 a$ (solid line). gap materials in regions where $\epsilon$ is frequency dependent.

In nonabsorbing cases, it is commonly accepted that the examined PBG "crystal" must be as thick as possible because the transmission inside the gap is thickness dependent. So, for the thicker "crystal," the transmission will be lower and the gap more well developed. In Fig. 7, the transmission vs the frequency is given for a PBG "crystal" with a "3-cylinder" structure consisting of holes in GaAs with filling ratio $f=0.221, a=0.5 \mu \mathrm{m}$, and three different thicknesses, $l=2 a, 4 a, 8 a$. At $\omega=300$ $\mathrm{THz}$, which is close to the lower edge of the gap, the transmission is $-5 \mathrm{~dB}$ for $l=2 a$ and $4 a$, but it is $-25 \mathrm{~dB}$ for $l=8 a$. At $\omega=320 \mathrm{THz}$, which is well inside the gap but still not affected by the absorption, the thickness dependence of the transmission is more obvious; in particular, the transmission is $-10,-30$, and $-60 \mathrm{~dB}$ for $l=2,4$, and $8 a$, respectively. These results support the previous statement for nonabsorbing PBG "crystals." But, above the upper edge of the gap ( $\omega=400 \mathrm{THz})$, the transmission is also thickness dependent because of the absorption; it is $-10,-20$, and $-30 \mathrm{~dB}$ for $l=2,4$, and 8, respectively. Assuming an experiment in which the background noise is $-40 \mathrm{~dB}$, the transmission at the upper edge of the gap cannot experimentally be distinguished from the noise; in contrast, for $l=2$ and $4 a$, the transmission at the lower edge of the gap can be well measured and distinguished from the noise. So, for absorbing PBG materials, the thicker slabs are not the best candidates for giving gaps in contrast with the nonabsorbing cases, where the thicker slabs give better photonic band gaps.

\section{CONCLUSIONS}

We have calculated the transmission coefficient versus the frequency of the incident electromagnetic (EM) waves propagating in photonic-band-gap (PBG) structures con- structed from dispersive and highly absorbing materials. In particular, we have studied cases where the polariton gap (PG), which was created from the coupling between the transverse optical phonons and the transverse EM waves and can be described from a frequency-dependent dielectric constant [Eq. (1)], is close to a structural gap, created from the periodicity of the lattice. In that case, for each gap of the frequency-independent case corresponds two ("twin") gaps in the frequency-dependent case, one above and one below the PG.

We have also studied how the absorption affects the structural gaps and the possible difficulties of their experimental investigation. We have found that, for absorbing cases, the transmission becomes thickness dependent for every frequency (for nonabsorbing cases, the transmission is basically thickness independent, except for frequencies inside the structural gaps). This thickness dependence increases as the frequency increases. As a consequence, for a very thick absorbing system, the transmission in the upper edge of a structural gap could become so small that it could be impossible to find the recovery of the transmission at the upper edge of the gap in an experiment where there will always be a lower bound for a transmission measurement (noise level). Thus, for absorbing PBG materials, the thicker slab is not necessarily better in contrast with what is commonly accepted for nonabsorbing PBG materials.

\section{ACKNOWLEDGMENTS}

Ames Laboratory is operated by the U.S. Department of Energy by Iowa State University under Contract No. W-7405-Eng-82. This work was supported by the director for Energy Research, Office of Basic Energy Sciences, including a grant of computer time on the Cray Computer at the Lawrence Livermore Laboratory and by NATO Grant No. RG769/87.

\footnotetext{
${ }^{1}$ See the special issue of J. Opt. Soc. Am. B 10, 208-408 (1993) on "Development and Applications of Materials Exhibiting Photonic Band Gaps."

${ }^{2}$ Photonic Band Gaps and Localization, edited by C. M.
}

Soukoulis, Proceedings of the NATO Advanced Science Institutes Series (Plenum, New York, 1993).

${ }^{3}$ K. M. Ho, C. T. Chan, and C. M. Soukoulis, Phys. Rev. Lett. 65, 3152 (1990). 
${ }^{4}$ C. T. Chan, K. M. Ho, and C. M. Soukoulis, Europhys. Lett. 16, 563 (1991).

${ }^{5}$ E. Yablonovitch, T. J. Gmitter, and K. M. Leung, Phys. Rev. Lett. 67, 2295 (1991).

${ }^{6}$ H. S. Sozuer, J. W. Haus, and R. Inguva, Phys. Rev. B 45, 13962 (1992); J. Opt. Soc. Am. B 10, 296 (1993).

${ }^{7}$ P. R. Villeneuve and M. Piche, Phys. Rev. B 46, 4969 (1992); 46, 4973 (1992).

${ }^{8}$ R. D. Meade, K. D. Brommer, A. M. Rappe, and J. D. Joannopoulos, Appl. Phys. Lett. 61, 495 (1992).

${ }^{9}$ M. Plihal, A. Shambrook, A. A. Maradudin, and P. Sheng, Opt. Commun. 80, 199 (1991); M. Plihal and A. A. Maradudin, Phys. Rev. B 44, 8565 (1991).

${ }^{10}$ E. Yablonovitch, T. J. Gmitter, R. D. Meade, A. M. Rappe, K. D. Brommer, and J. D. Joannopoulos, Phys. Rev. Lett. 67, 3380 (1991).
${ }^{11}$ R. D. Meade, K. D. Brommer, A. M. Rappe, and J. D. Joannopoulos, Phys. Rev. B 44, 13772 (1991).

${ }^{12}$ S. L. McCall, P. M. Platzman, R. Dalichaouch, D. Smith, and S. Schultz, Phys. Rev. Lett. 67, 2017 (1991).

${ }^{13}$ W. Robertson, G. Arjavalingan, R. D. Meade, K. D. Brommer, A. M. Rappe, and J. D. Joannopoulos, Phys. Rev. Lett. 68, 2023 (1992).

${ }^{14}$ J. B. Pendry and A. MacKinnon, Phys. Rev. Lett. 69, 2772 (1992); J. B. Pendry, J. Mod. Opt. (to be published).

${ }^{15}$ M. Sigalas, C. M. Soukoulis, E. N. Economou, C. T. Chan, and K. M. Ho, Phys. Rev. B 48, 14121 (1993).

${ }^{16} \mathrm{E}$. Yablonovitch (private communication).

${ }^{17}$ C. Kittel, Introduction to Solid State Physics (Wiley, New York, 1976).

${ }^{18}$ E. D. Palick, in Handbook of Optical Constants of Solids, edited by E. D. Palik (Academic, London, 1985), p. 434. 